\title{
Thiolated and PEGylated silica nanoparticle delivery to hair follicles
}

\section{Article}

Accepted Version

Creative Commons: Attribution-Noncommercial-No Derivative Works 4.0

Al Mahrooqi, J. H., Khutoryanskiy, V. V. and Williams, A. ORCID: https://orcid.org/0000-0003-3654-7916 (2021)

Thiolated and PEGylated silica nanoparticle delivery to hair follicles. International Journal of Pharmaceutics, 593. 120130. ISSN 0378-5173 doi: https://doi.org/10.1016/j.ijpharm.2020.120130 Available at https://centaur.reading.ac.uk/94775/

It is advisable to refer to the publisher's version if you intend to cite from the work. See Guidance on citing.

To link to this article DOI: http://dx.doi.org/10.1016/j.ijpharm.2020.120130

Publisher: Elsevier

All outputs in CentAUR are protected by Intellectual Property Rights law, including copyright law. Copyright and IPR is retained by the creators or other copyright holders. Terms and conditions for use of this material are defined in the End User Agreement.

\section{www.reading.ac.uk/centaur}

\section{CentAUR}


Central Archive at the University of Reading

Reading's research outputs online 


\section{Thiolated and PEGylated silica nanoparticle delivery to hair follicles}

Jamila H. Al Mahrooqi, Vitaliy V. Khutoryanskiy, Adrian C. Williams*

Reading School of Pharmacy, University of Reading, Reading RG6 6AD, U.K.

a.c.williams@reading.ac.uk

Abstract:

Targeting drug delivery to hair follicles is valuable to treat conditions such as alopecia's and acne, and this shunt route may also allow drug delivery to deeper skin layers and the systemic circulation by avoiding the intact stratum corneum. Here, we investigated the effects of nanoparticle surface chemistry on their delivery into hair follicles by synthesizing fluorescent thiolated silica nanoparticles and functionalizing with $750 \mathrm{Da}$ and $5000 \mathrm{Da}$ methoxypolyethylene glycol maleimide (PEG). The stability of the nanoparticles in skin homogenate was verified before tape stripping of porcine-dosed tissue showed the distribution of the free fluorescent dye and different nanoparticles in the skin. Analysis of microscopic images of the skin sections revealed penetration of nanoparticles functionalized with PEG into the appendages whereas thiolated nanoparticles stayed on the surface of the skin and were removed by tape stripping. Nanoparticles functionalized with PEG 5000 Da penetrated deeper into the hair follicles compared to counterparts functionalized with PEG 750 Da. PEGylation can thus enhance targeted delivery of nanoparticulates into hair follicles.

Keywords: follicular targeting; nanoparticles; silica; PEGylation; skin 
The remarkable barrier properties of human skin are largely attributed to the outermost layer of the epidermis, the stratum corneum (Brown and Williams, 2019; Labouta and Schneider, 2013). Appendages including the hair follicles and eccrine glands cross the epidermis and originate in the underlying dermis. Given the stratum corneum barrier, it is well established that typically only relatively small molecular weight and lipophilic drugs are able to be delivered effectively via the transdermal route to clinically efficacious levels, though some recent studies have shown that macromolecules such as aptamers may be able to enter the tissue (Lenn et al., 2018). However, the use of nanoparticles for drug delivery into or through the skin has also been studied using quantum dots, carbon nanotubes, metal and metal oxide nanoparticles, dendrimers, and lipid based nanoparticles (Jensen et al., 2011; Kraeling et al., 2018; Küchler et al., 2009).

Nanoparticulate dosing of skin tends to focus on two mechanisms of delivery. Firstly, the potential delivery of the nanosystem into intact stratum corneum (and deeper epidermal layers) with studies exploring the effects of physicochemical properties of the nanosystems including their size, shape, charges and surface properties (Labouta et al., 2011a; Labouta and Schneider, 2013; Lademann et al., 2011). Other factors considered include the nanoparticle vehicle and the disease state of the skin or stratum corneum barrier impairment (for example during wound healing) (Brown and Williams, 2019; Larese et al., 2009; Vogt et al., 2016; Wu et al., 2009). Secondly, the nanoparticles may be delivered into the appendages (pilosebaceous unit and eccrine and apocrine ducts). Though varying from site to site (Otberg et al., 2004) the appendage openings represent approximately $0.1 \%$ of the skin surface area and provides a shunt route to the lower layers of the skin by circumventing the intact surface stratum corneum (Brown and Williams, 2019; Raber et al., 2014). The follicles have been targeted with nanoparticles to treat conditions such as alopecia and acne, and alternatively to deposit a reservoir of a drug to permeate into deeper skin layers or the systemic circulation.

Particle deposition into a hair follicle is usually stimulated in vivo by hair movement and (as with in vitro studies) by massage. Further, the cuticles on the surface of the hair shaft form a zigzag structure which acts as a geared pump that, with hair movement, can push nanoparticles deeper into the hair follicles. Clearance of nanoparticles from the follicle depends primarily on sebum production and hair growth, both relatively slow processes allowing the follicle to serve as a reservoir; when particles were applied, their clearance from the hair follicle was over a period of 10 days (Knorr et al., 2009; Lademann et al., 2009; Raber et al., 2014). More specifically within the hair follicle, the infundibulum generally provides the reservoir compartment whereas sebaceous glands are associated with acne and androgenetic alopecia and the bulge region holds a reservoir for keratinocyte stem cells (Knorr et al., 2009; Patzelt et al., 2011). 
However, the optimal nanoparticle properties for deposition into hair follicles are unclear and variations are also seen with delivery vehicle; Mathes et al. reported greater follicular deposition of particles from aqueous dispersions compared to when suspended in a hydrogel and that follicular uptake can thus be controlled by the choice of vehicle or carrier used to deliver a drug (Mathes et al., 2016). Patzelt et al. (2011) compared the depth of penetration into hair follicles of different sizes $(122,230,300,470,643$, and $860 \mathrm{~nm}$ ) of two types of nanoparticles, PLGA (polylactide-co-glycolid) and silica. Both types showed similar size-dependent penetration, increasing in depth as size rose to $643 \mathrm{~nm}$, but the silica nanoparticles penetrated deeper than the PLGA particles. It had been proposed that the geared pump effect from hair shaft movement is optimal when the particles are similar size to the hair cuticles - in the region of $500 \mathrm{~nm}$ (Lademann et al., 2009; Patzelt et al., 2011). However, Vogt et al. reported deeper penetration of $40 \mathrm{~nm}$ nanoparticles along the follicular duct of human vellus hair and through the follicular epithelium, whereas 750 and $1500 \mathrm{~nm}$ nanoparticles aggregated in the infundibulum with no penetration to deeper layers or through viable epidermis (Vogt et al., 2006).

Varied nano-particulate systems have been evaluated for controlled drug delivery within hair follicles, ranging from liposomes and polymersomes to solid lipid nanoparticles and nano-vesicles. Commonly seeking to treat alopecias, examples include oleic acid and phosphatidylcholine nano-vesicles containing minoxidil which increased deposition of the drug in the follicles 10-fold compared to a gel formulation (Kumar et al., 2018). Minoxidil has also been incorporated into liposomes (e.g. Jain et al., 2010), solid lipid nanoparticles (Padois et al., 2011) and into nanoparticles formed from PLGA (Takeuchi et al., 2018) - as has finasteride (Roque et al., 2017). The use of nano-sized delivery systems to target hair follicles in alopecia, along with consideration of the anatomy and pathophysiology of the condition, has recently been reviewed (Salim and Kamalasanan, 2020). Nanocarriers have also been used for glucocorticoids such as clobetasol propionate for inflammatory scalp conditions. To mitigate both systemic and local adverse effects when applied topically, a range of clobetasol-loaded polymeric nanocarriers were evaluated (nanospheres, nanocapsules, lipid-core nanocapsules) to balance drug release, interfollicular permeation and follicular uptake (Schiedel et al., 2015). Significantly greater levels of drug were found in the follicles from the nanocarriers compared to application of the free drug and, importantly, the study demonstrated that not only was the carrier important but also that massaging the tissue resulted in greater follicular uptake, in agreement with the above.

PEGylation is widely used to improve stability, tolerance and delivery to various tissues including eyes, urinary bladder, and nasal tissue (Porfiryeva et al., 2020). We have previously shown that PEGylation of nanoparticles reduces their mucoadhesion and improves their diffusivity (Irmukhametova et al., 2011; Mun et al., 2014b; Mun et al., 2016; Ways et al., 2018). However, nanoparticles with thiol groups on their surface are expected to stay bound to the skin surface and hair as they are rich in keratin. Polyacrylic acid was modified with sulfhydryl moieties to improve its binding to the skin, resulting in a 15-fold increase in adhesion compared to unmodified polymer (Laffleur and Bernkop-Schnürch, 2018). Likewise, thiolation of silicone oil enhanced adhesion to skin in comparison to commonly used silicone oils (Partenhauser et al., 2016). Here, we used tape 
striping to study the follicular deposition of two types of silica nanoparticles with different surface chemistry and sizes, thiolated and PEGylated (750 and $5000 \mathrm{Da}$ ) silica nanoparticles. The particles were fluorescently labelled with 5iodoacetamido fluorescein and follicular uptake was investigated in vitro using pig flank skin.

2 Material and methods:

\subsection{Materials:}

3-mercaptopropyltrimethoxysilane (MPTS 95\%), maleimide terminated methoxy poly(ethylene glycol) (PEG molecular weight 750 \& $5000 \mathrm{Da}$ ), sodium phosphate dibasic $(\geq 99 \%)$, sodium phosphate monobasic dihydrate $(\geq 99 \%)$, 5(iodoacetamido)fluorescein, sodium fluorescein, and 5,5'-dithiobis(2nitrobenzoic acid) (DTNB, $\geq 98 \%$ TLC), were purchased from Sigma-Aldrich (UK). DMSO, sodium hydroxide pellets and Slide-A-Lyzer minidialysis devices, 3.5kDa MWCO, were purchased from Fisher Scientific (UK). L-Cysteine hydrochloride anhydrous (98\%) was purchased from Alfa Aesar (UK). Dialysis membrane with a molecular weight cut-off 12-14 kDa was purchased from Medicell International Ltd. (UK). D-Squame Standard Sampling Discs and D- Squame $\AA$ disc applicator was purchased from Clinical and Derm (USA). All materials were used as received. Fresh newborn ( $<1$ month old) pig flank skin was obtained from the Pirbright Institute (UK) and was frozen immediately after collection until use.

\subsection{Synthesis and characterization of thiolated silica nanoparticles:}

Thiolated silica nanoparticles were synthesised according to a previously published method (Irmukhametova et al., 2011). Briefly, $20 \mathrm{~mL}$ DMSO and 0.5 $\mathrm{mL}$ of $0.5 \mathrm{M} \mathrm{NaOH}$ solution were added to $0.75 \mathrm{~mL}$ MPTS. The mixture was stirred continuously with air bubbling for $24 \mathrm{~h}$ at room temperature. The nanoparticles were purified by dialysis against deionized water $(5 \mathrm{~L}$, eight changes of water) using dialysis membrane. The purified aqueous dispersions of the nanoparticles were stored at $4{ }^{\circ} \mathrm{C}$.

2.3 Synthesis of fluorescently-labelled thiolated silica nanoparticles:

Thiolated silica nanoparticles were labelled with 5-(iodoacetamido) fluorescein (5-IAF) by adding $3 \mathrm{mg}$ of 5-IAF to $12 \mathrm{~mL}$ aqueous dispersions of thiolated nanoparticles. The amount of fluorophore used was calculated with respect to molar ratio so that $5 \mu \mathrm{mol}$ of fluorophore is added to $50 \mu \mathrm{mol}$ of sulfhydryl groups of nanoparticles. The reaction mixture was stirred for $16 \mathrm{~h}$ at room temperature protected from light. Fluorescently-labelled nanoparticles were then purified by dialysis against deionized water in the dark ( $5 \mathrm{~L}$, eight changes of water), according to the above protocol (Irmukhametova et al., 2011).

\subsection{PEGylation of fluorescently-labelled nanoparticles:}

$5 \mathrm{~mL}$ aqueous dispersions of fluorescently-labelled nanoparticles were mixed 
with $100 \mathrm{mg}$ of methoxypolyethylene glycol maleimide of two molecular weights (750 or $5000 \mathrm{Da}$ ). The reaction mixture was stirred for $16 \mathrm{~h}$ at room temperature protected from light, resulting in the formation of PEGylated silica nanoparticles. PEGylated nanoparticles were purified by dialysis in the dark as above (Mun et al., 2014b).

\subsection{Characterization of nanoparticles}

DLS and 3-potential measurements were conducted with dilute dispersions of nanoparticles at $25{ }^{\circ} \mathrm{C}$ using a Nano-S Zetasizer (Malvern Instruments, UK). Each batch of nanoparticles was synthesized in triplicate, and the analysis was carried out three times for each sample. The mean and standard deviation of particle size, polydispersity, and 3-potential were calculated. The thiol group content in nanoparticles was determined by Ellman's assay according to a previously published method (Al Mahrooqi et al., 2018).

\subsection{Stability study}

The stability of the fluorescent label on the nanoparticles for use in skin studies was evaluated by adding the nanoparticles to skin homogenate and dialysing against phosphate buffered saline (PBS) to assess the release of free dye. Briefly, $2 \mathrm{~g}$ of pig skin was cut into small pieces and homogenised in $15 \mathrm{~mL}$ of PBS at 6500 rpm for 1 minute in an ice bath using an Ultra Turrax T18 high speed homogenizer (Lau et al., 2013). Four different samples were prepared by mixing $1.5 \mathrm{~mL}$ of the labelled nanoparticles, unlabelled nanoparticles, sodium fluorescein and homogenate respectively with $1.5 \mathrm{~mL}$ of homogenate (total $3 \mathrm{~mL}$ ) and dialysed against PBS. The samples were placed on a rolling plate with continuous shaking at $37^{\circ} \mathrm{C}$ to ensure continuous mixing and movement of the dialysate. The fluorescence intensity of the dialysate was assessed using a fluorescent spectrophotometer after 1, 6, 24, 48, and 72 hours of incubation. The experiment was performed in triplicate and data were analysed using GraphPad Prism 8 software.

\subsection{Tape stripping study}

Pig flank skin was thawed at room temperature and hair was carefully shaved to avoid damaging the stratum corneum before use. Test areas were demarked using a permanent marker around a $2.5 \times 2.5 \mathrm{~cm}$ polyethylene template. A finite dose of fluorescently labelled nanoparticles ( $50 \mu \mathrm{L}$, so $\left.8 \mu \mathrm{L} \mathrm{cm}{ }^{-2}\right)$ was applied and the area gently massaged in a circular movement for one minute using a gloved fingertip. The dosed tissue was then left for 2 hours at room temperature, protected from light. D-Squame ${ }^{\circledR}$ adhesive discs were used for tape stripping; each disc was pressed firmly against the skin using a $D$ - Squame ${ }^{\circledR}$ disc applicator with a constant pressure of $225 \mathrm{~g} / \mathrm{cm}^{2}$ for $5 \mathrm{sec}$. The marked area was stripped sequentially twenty times with the direction of stripping alternating for each strip. After each strip, both the adhesive disc containing the removed layer of stratum corneum and the remaining skin sample were analysed using a Leica MZ10 F stereomicroscope (Leica Microsystems, UK) with an ET-GFP filter to 
quantify the presence of fluorescent nanoparticles and show their distribution. All images were taken with an exposure time of $80 \mathrm{mS}$, gain 2, gamma 1 and wavelength $520 \mathrm{~nm}$. Images were further analysed using ImageJ software (National Institutes of Health, USA) and normalised with the following equation:

\section{Fluorescence intensity $=\frac{I-I_{b}}{I_{0}-I_{b}} \times 100$ equation 1}

where $I$ is the fluorescence intensity of the nanoparticle-dosed skin sample or disc after each tape stripping and $l_{0}$ is the initial fluorescence intensity of the sample following dosing, massage and incubation but prior to any tape stripping. $I_{b}$ is the background fluorescence intensity of the disc or skin; to allow each individual tissue sample to act as its own background fluorescence control (Ways et al., 2018), and recognising that this may change the sequential removal of the stratum corneum, background fluorescence was measured after each tape stripping of non-dosed skin samples using the same protocol as above.

In addition, each adhesive disc was weighed before and after tape stripping in order to determine the fraction of the barrier removed or relative depth $(x / L)$; where $\mathrm{x}$ is the thickness removed divided by the total stratum corneum thickness $(\mathrm{L})$ and so relative depth within the stratum corneum varies between 0 (at the skin surface) to 1 at the interface with the viable epidermis (Russell and Guy, 2012). Each disc was then centrifuged in $5 \mathrm{~mL}$ PBS at $3500 \mathrm{rpm}$ for 10 minutes before the fluorescence intensity of the extract was assayed using a fluorescence spectrophotometer (Cary Eclipse, Varian Inc., US) at $494 \mathrm{~nm}$ excitation and an emission spectral range of $500-600 \mathrm{~nm}$.

The tape stripped skin area was then excised and placed on a weighing boat with the epidermis facing upwards and fully covered with OCT embedding medium and placed in dry ice. The frozen sample was stored at $-80^{\circ} \mathrm{C}$ before tissue cross sectioning at $-20^{\circ} \mathrm{C}$ using a Bright 5040 cryostat microtome (Bright Instruments Ltd., Luton, UK). Tissue was placed perpendicularly to the cutting blade (i.e. tissue cut from dermis to outer surface) to avoid dislocation of the nanoparticles from outside into deeper skin layer (Labouta et al., 2011a, 2011b). Crosssections $(10 \mu \mathrm{m})$ were placed on microscopic slides and imaged using a Zeiss Axio Imager fluorescence microscope (Carl Zeiss, Jena, Germany). The samples were excited with a $488 \mathrm{~nm}$ filter in order to locate the labelled nanoparticles and with a DAPI filter to view the tissue. The two images were merged using Carl Zeiss software and analysed by ImageJ software.

3 Results and discussion:

3.1 Synthesis and characterization:

Thiolated silica nanoparticle were synthesized by hydrolysis and selfcondensation of 3-mercaptopropyltrimethoxysilane (MPTS) in the aprotic solvent dimethyl sulfoxide (DMSO) in contact with air, with $\mathrm{NaOH}$ as the catalyst. Sub$100 \mathrm{~nm}$ cross-linked nanoparticles are formed through Si-O-Si and disulfide bonds. The nanoparticles were labelled with 5-IAF, where the iodoacetamide moiety of the fluorophore reacts with the sulfhydryl group in the nanoparticles. 
Fluorescently labelled nanoparticles were then PEGylated and both labelled thiolated and PEGylated silica nanoparticles were used in all experiments. It has been reported that a high grafting density of lower molecular weight PEG and decorating particles with PEG 5000 Da provide rapid mucus penetrating properties, whereas, nanoparticles became mucoadhesive when coated with PEG 10000 Da (Wang et al., 2008). Therefore, PEG 750 and 5000 Da were chosen for our experiments.

The resulting nanoparticles significantly $(P<0.05)$ increased in size in the order thiolated $<$ PEGylated $750 \mathrm{Da}<$ PEGylated $5000 \mathrm{Da}$ and they all had a narrow polydispersity index (PDI; Table 1 and Figure S1) and negative 3-potential values due to the presence of thiol groups at their surface. Following PEGylation, the nanoparticles' 3-potential values reduced due to the polymer coupling with some of the free thiol on the surface of the nanoparticles, with the greater decrease seen for the larger molecular weight PEG also partially attributed to some charge shielding effects. Indeed, the Ellman's assay showed a statistically significant $(P<0.005)$ difference in free thiol content between thiolated and the PEGylated nanoparticles, and no significant difference between PEGylated 750 and 5000 Da particles. The reduction in thiol content and increase in particle size indicates successful PEGylation of the nanoparticles (Table 1), but clearly these particles retain some free thiol groups at their surface - approximately $50 \%$ of those seen in the parent thiolated material. Nanoparticle concentrations were determined by freeze-drying $1 \mathrm{~mL}$ of nanoparticle suspensions and determining the weight of the solid residue. This concentration was used to calculate free thiol content before and after PEGylation.

\begin{tabular}{|l|l|l|l|l|l|}
\hline Nanoparticle & $\begin{array}{l}\text { Size } \\
(\mathbf{n m})\end{array}$ & PDI & $\begin{array}{l}\text { 3-potential } \\
(\mathbf{m V})\end{array}$ & $\begin{array}{l}\text { Concentration } \\
(\mathbf{m g} / \mathbf{m L})\end{array}$ & $\begin{array}{l}\text { Free thiol content } \\
(\boldsymbol{\mu m o l} / \mathbf{g})^{\star}\end{array}$ \\
\hline Thiolated & $62 \pm 3$ & $0.207 \pm 0.015$ & $-47 \pm 3$ & $15 \pm 1$ & $289 \pm 14$ \\
\hline PEGylated 750 Da & $79 \pm 1$ & $0.214 \pm 0.011$ & $-42 \pm 3$ & $12 \pm 1$ & $144 \pm 21$ \\
\hline PEGylated 5000 Da & $89 \pm 1$ & $0.169 \pm 0.010$ & $-30 \pm 2$ & $20 \pm 2$ & $142 \pm 2$ \\
\hline
\end{tabular}

Table 1: Characterization of thiolated and PEGylated silica nanoparticles, $n=3$, values presented as mean \pm standard deviation. *The calibration curve used to calculate free thiol content is shown in Figure S2

\subsection{Stability of nanoparticles in skin homogenate:}

The skin possesses both enzymatic and non-enzymatic detoxification systems including glutathione (GSH) as an antioxidant (Connor and Wheeler, 1987) which acts as a direct free radical scavenger and quenches radicals by hydrogen atom donation resulting in the formation of glutathione disulphide (GSSG). However, as the reduced form of GSH is essential for cutaneous photo-protection, GSSG is recycled to GSH by GSH-reductase with hydrogen donation by nicotinamide adenine dinucleotide phosphate (NADPH). This process is extremely fast due to the high activity of the enzyme and it takes less than one minute to restore all cutaneous GSH (Connor and Wheeler, 1987). It was anticipated that nanomaterials with disulfide bonds are susceptible to degradation by GSH (Cui et al., 2012; Hayashi et al., 2016; Prasetyanto et al., 2016; Yang et al., 2016; Zhang et al., 2012; Zhou et al., 2017), and so the stability of the fluorescent label attached to the nanoparticle core before further decoration with PEG was investigated in skin 
homogenate. The release of fluorescent dye from the fluorescent thiolated silica nanoparticles was examined in the phosphate buffer ("labelled TSNP's") and in skin homogenate, alongside assays of sodium fluorescein with skin homogenate as a positive control and the homogenate alone to detect auto-fluorescence; the homogenate showed no detectable auto-fluorescence during the study. The release of the fluorophore was measured over time (Figure 1) and sodium fluorescein was detected in the dialysate at 6 hours $(4 \%)$ and reached $(11 \%)$ at 72 hours. The labelled particles showed a similar release profile when incubated in either buffer of skin homogenate with very low fluorescence first detected after 24 hours and no significant differences in release in these two media $(p>0.05)$. Release of sodium fluorescein across the dialysis membrane from the positive control was significantly greater than release from the nanoparticles $(p<0.05)$.

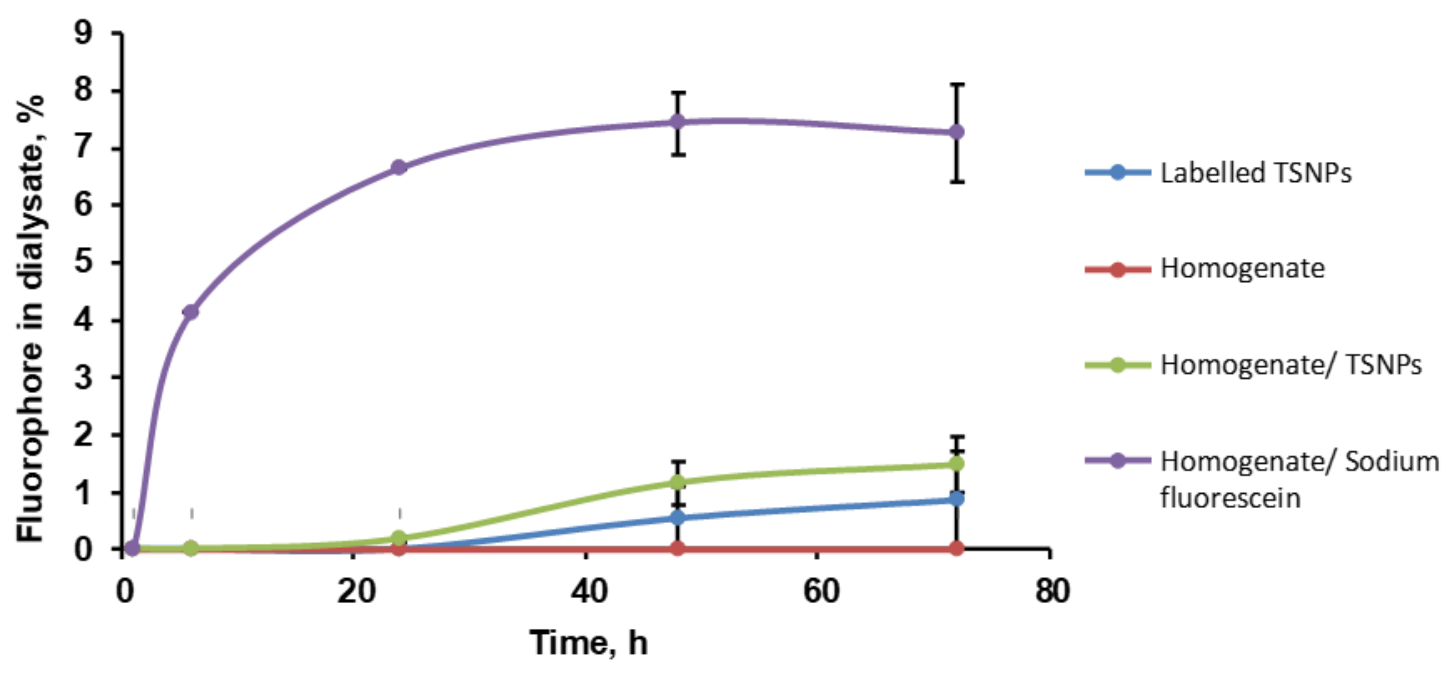

Figure 1: The release of fluorescent dye across the dialysis membrane. Labelled TSNP = fluorophore release from labelled silica nanoparticles in buffer; Homogenate = homogenate alone to test for autofluorescence; Homogenate/TSNP's = fluorophore release from labelled silica nanoparticles in skin homogenate; Homogenate/Sodium fluorescein is the positive control of the fluorescent dye with homogenate. Study at $37^{\circ} \mathrm{C}$, samples taken at 1, 6, 24, 48, 72 hours, $n=3$, values are presented as mean \pm standard error of mean.

Doura et al. (2019) demonstrated that MPTS nanoparticles were resistant to GSHinduced degradation and retained their spherical shape. In their experiments, they prepared various hybrid thiol-organosilica nanoparticles from different proportions of 3-mercaptopropyltrimethoxysilane (MPTS) and 3mercaptopropyldimethoxymethylsilane (MPDMS) and detected the ratio of disulphide bonds to thiol groups in each sample by Raman spectroscopy. They investigated the degradability of the nanoparticles in 10 and $40 \mathrm{mM}$ of GSH, compared with nanoparticles prepared from MPTS or MPDMS alone. They found that increasing the ratio of MPDMS increased the susceptibility to degradation by GSH. They also found that the introduction of MPDMS into nanoparticles prepared from MPTS induced the formation of disulfide bonds which was proportional to the ratio of MPDMS constituent (Doura et al., 2019). In our study, the particles produced from MPTS likewise showed no significant degradation in the skin homogenate. Further, both labeled and unlabeled nanoparticles were stored for up to 6 months and no flocculation or aggregation was observed, again in agreement with previous studies using similar nanoparticles (Ways et al., 2018). 


\subsection{Tape stripping study:}

Equation 1 was used to quantify the fluorescence intensity of the skin and tape strips, and relies on subtraction of background fluorescence $\left(I_{b}\right)$. Prior to dosing, we investigated the effects or tape stripping skin on the background fluorescence of the tissue. The experiments (in triplicate) showed a gradual reduction in the background intensity with tape stripping such that after 20 strips, the background intensity had fallen by a mean of $12 \%$. These values were used for the background intensities in subsequent dosed studies.

Tape stripping is increasingly used for bioavailability and bioequivalence studies (Herkenne et al., 2008; Pensado et al., 2019). However, specific study protocols vary, for example in cutting or shortening the hair prior to experimentation (Jensen et al., 2011; Klang et al., 2011; Nagelreiter et al., 2015; Patzelt et al., 2011). Here, the hair was shaved since preliminary studies showed that our nanoparticles bound strongly to shortened hair bristles even after 20 tape stripping (Figure S3 a) but binding was reduced with PEGylated nanoparticles (Figure S3 b \&c). Keratin is rich in disulfide bonds and thiolated silica nanoparticles interact covalently with hair keratin (Bragulla and Homberger, 2009; Cruz et al., 2017; Gniadecka et al., 1998; Williams et al., 1994). PEGylation of the thiolated particles reduces the free thiol groups in the particle shell by about 50\% (Table1) and may also screen remaining thiol groups on the particle surface. A similar trend with mucoadhesion to different tissues was reported where mucoadhesive properties of the thiolated silica nanoparticles were significantly decreased by PEGylation and penetration was improved (Irmukhametova et al., 2011; Mun et al., 2014b; Ways et al., 2018).

Significant reductions $(P<0.05)$ in fluorescence intensity of all tested nanoparticles and sodium fluorescein was evident after 2 hours of incubation on the skin surface and prior to tape stripping. The fluorescence intensity reduced to $12,52,7$ and $10 \%$ for sodium fluorescein, TSNPs, PEGylated 750 Da and PEGylated 5000 Da TSNPs respectively which could be attributed to quenching of fluorescein (Glasgow, 2016; Song et al., 1995) and penetration into the follicles. Teichmann et al. reported recovery of $95 \%$ of sodium fluorescein dye from stratum corneum and $5 \%$ from follicular infundibula after differential stripping (Teichmann et al., 2005). The penetration of sodium fluorescein solution into stratum corneum has also been compared with that when loaded into PEGylated lipid nanocarriers (Rangsimawong et al., 2016). In another study, Zhang et. al. used transmission electron microscopy to study PEG-coated quantum dots applied to skin and reported localization within the lipid bilayers of the stratum corneum. This was attributed to the soft coating of PEG which enabled the quantum dots to enter the intercellular lipid matrix of the stratum corneum although this is unlikely the case for our larger PEGylated nanoparticles (Zhang et al., 2008). It is notable that a far larger proportion of the fluorescence remained at the skin surface 2 hours post-dosing of the thiolated silica nanoparticles (prior to tape stripping). Fourier transform Raman and infrared vibrational analysis of the stratum corneum demonstrated the presence of disulphide bonds in proteins (Barry et al., 1992; Gniadecka et al., 1998) and the keratins that form the stratum corneum are relatively soft and contain little cysteine compared to hair (Williams et al., 1994). Retention of the thiolated silica nanoparticles on the skin surface is thus likely attributable to the formation of 
disulphide bonds with the outer hair shaft and, to a lesser extent, stratum corneum keratins.

We used spectroscopic and gravimetric analysis to generate nanoparticle distribution profiles as a function of stratum corneum depth (Figure S4). By assuming that the density of stratum corneum and area stripped are constant and knowing the weight of stratum corneum removed, the mass of sample removed can be related to tissue depth (Higo et al., 1993). The distribution profiles only showed significant differences between thiolated silica nanoparticles and sodium fluorescein $(p<0.05)$ and no significant differences between either of the PEGylated particles or the thiolated material (Figure 2). Sodium fluorescein has been reported to readily penetrate into the stratum corneum but less well into the deeper layers of the epidermis (Rangsimawong et al., 2016). Here, we see a reproducible penetration gradient down to approximately $60 \%$ of the stratum corneum thickness for the free dye (Figure 2). In contrast, there is no strong evidence for penetration of any of the nanoparticles into the intact stratum corneum with almost all particles removed by the second tape strip. Some fluorescence was seen but is attributed to particles remaining bound to the surface of the skin, held in skin furrows or adhered to the upper hair follicle and so were not removed by tape strips (Figure S4).

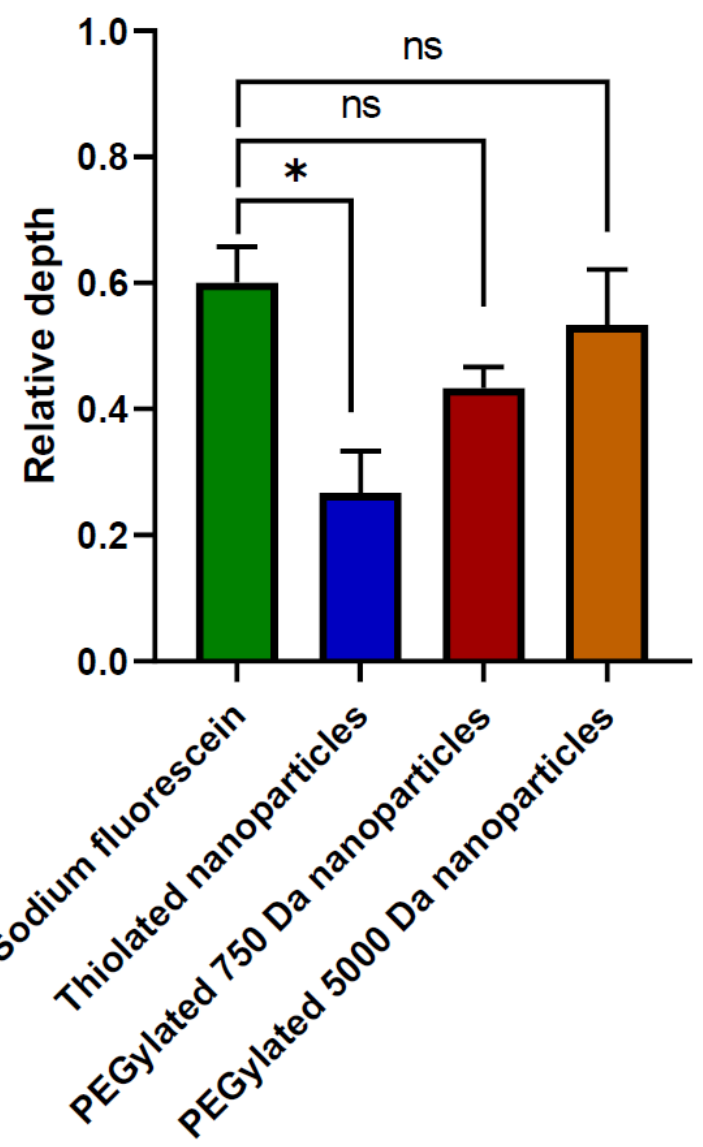

Figure 2: Penetration depth of sodium fluorescein, TSNPs, PEGylated (750 \& $5000 \mathrm{Da})$ TSNPs into the removed stratum corneum layers, $n=3, P<0.05$, values are presented mean \pm standard error of mean calculated using one-way ANOVA and Bonferroni post-hoc tests

Fluorescent microscope images of skin sections were taken to visualize the location 
of nanoparticles and clearly demonstrated their binding to hair follicles (Figure 3). The thiolated silica nanoparticles (Figure 3a) can be seen to adhere to the skin surface and external surface of the hair follicle as well as reside in a skin furrow. The images show no evidence for penetration into the deeper skin layers, in agreement with the results obtained from the tape strips. Sodium fluorescein penetrated into the hair follicles and analysis of the skin section images revealed a mean maximal penetration depth of 700 $\mu \mathrm{m}$ (Figure 3b). PEGylated nanoparticles penetrated into the hair follicles to a mean maximal depth of $450 \mu \mathrm{m}$ and $1400 \mu \mathrm{m}$ for PEGylated $750 \mathrm{Da}$ (Figure 3c) and 5000 Da (Figure 3d) nanoparticles respectively.

a

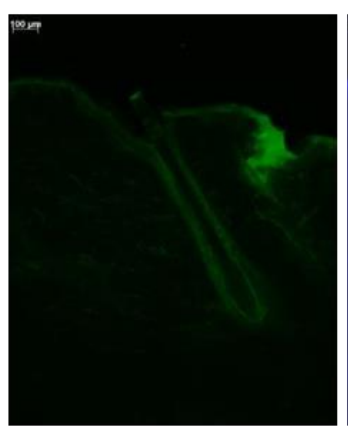

C

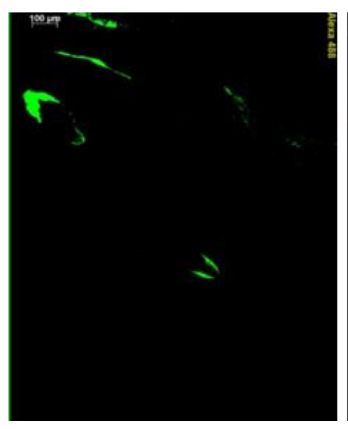

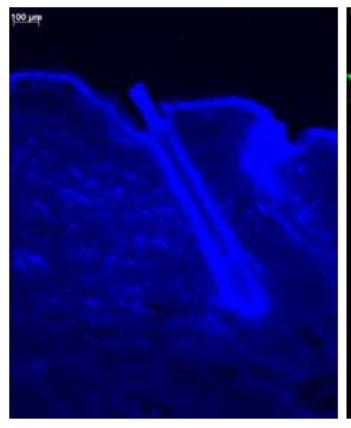
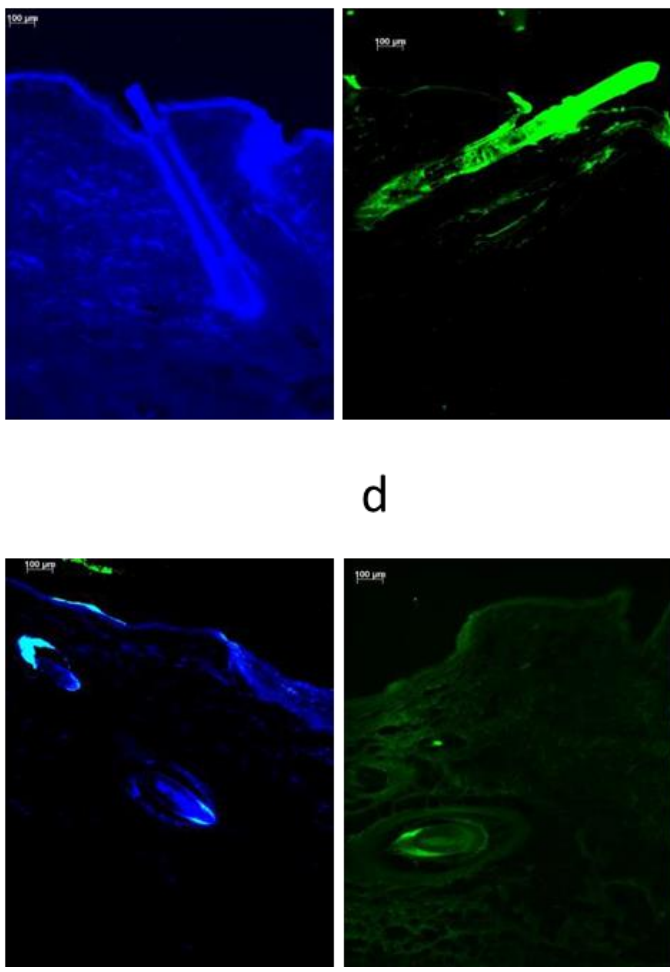

d

b

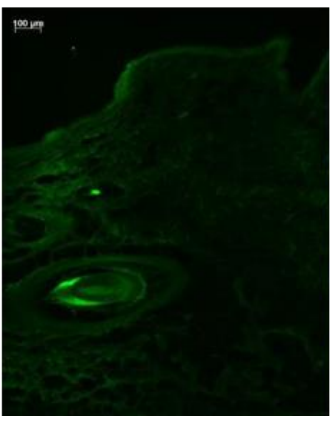

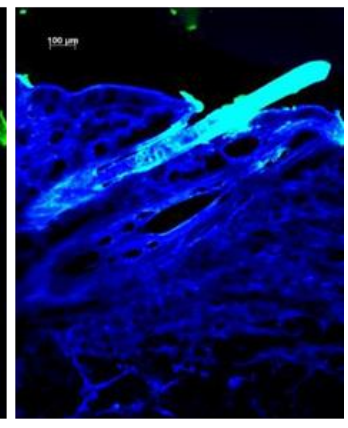

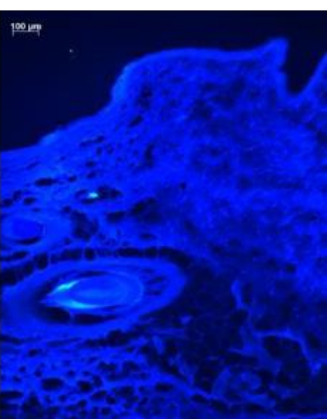

Figure 3: Exemplar fluorescence microscope images of tape stripped pig skin sections (10 $\mu \mathrm{m}$ thick) after application of thiolated nanoparticles (a), sodium fluorescein (b), PEGylated 750 Da nanoparticles (c) and PEGylated 5000 Da nanoparticles (d). For each set, the first image uses an Alexa $488 \mathrm{~nm}$ (green) filter to show only the nanoparticles and the second image merges this with a DAPI (blue) filter to visualise the nanoparticles with the skin background. Scale bar $100 \mu \mathrm{m}$.

Analysis of the images (12 images for each sample with three measurements for each image) revealed significant differences in particle penetration into the hair follicles with PEGylated 5000 Da nanoparticles penetrating deepest followed by sodium fluorescein, PEGylated $750 \mathrm{Da}$ nanoparticles and the fully thiolated materials penetrated least into the follicle $(p<0.0005)$ (Figure 4$)$. 


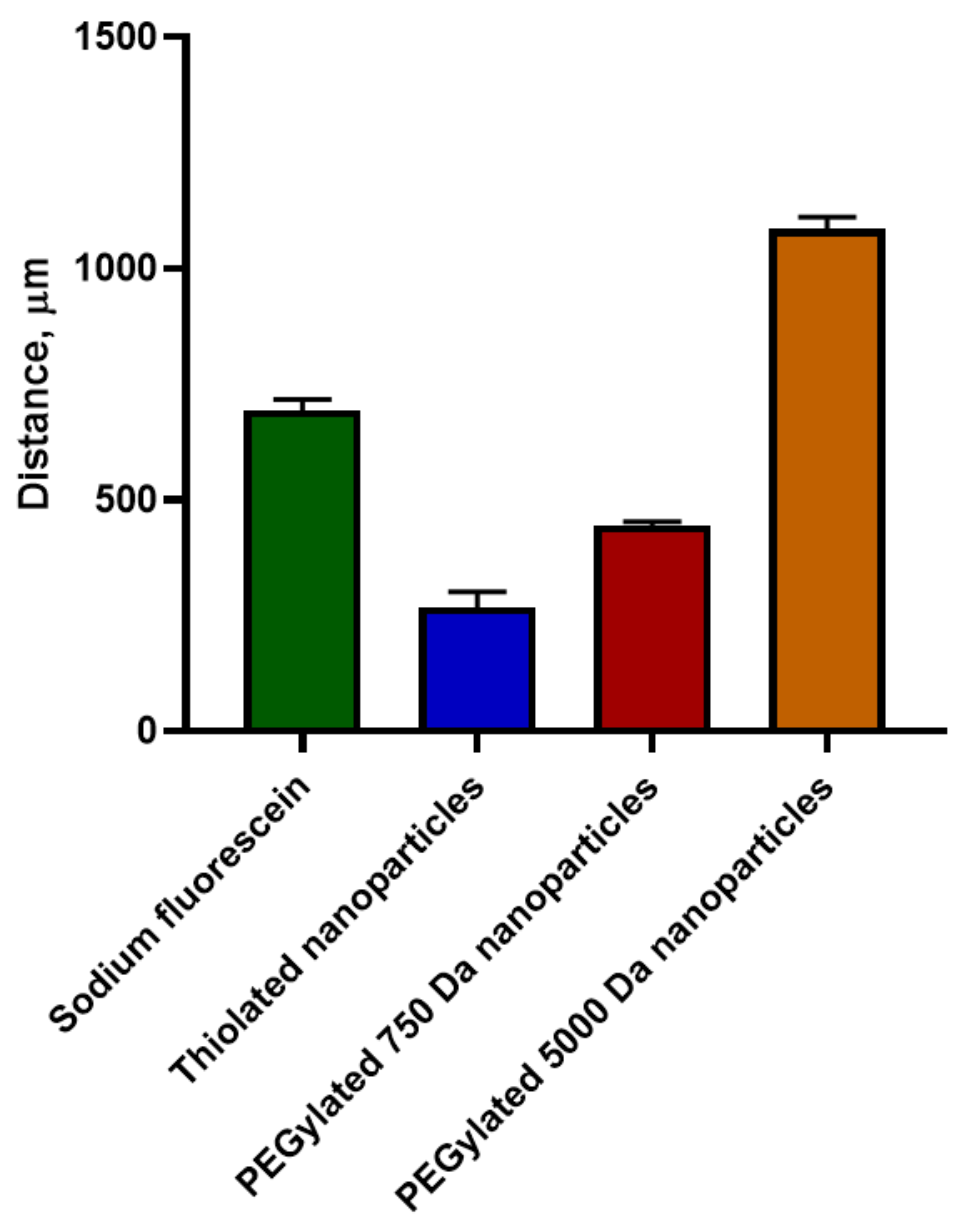

Figure 4: Depth of penetration into the hair follicles, $n=12$, all results are significantly different from each other $(P<0.0005)$, values are presented mean \pm standard error of mean calculated using one-way ANOVA and Bonferroni post-hoc tests.

Sodium fluorescein is a low molecular weight compound (376 Da) that was shown above to penetrate into the stratum corneum, in accord with literature reports. This water-soluble dye was also reproducibly seen at a depth of $700 \mu \mathrm{m}$ within the follicles, in the infundibulum and in the region of the sebaceous gland; Knorr et al. and Patzelt et. al. reported that the sebaceous gland is located $\sim 600 \mu \mathrm{m}$ to $900 \mu \mathrm{m}$ deep within the terminal hair follicle (Knorr et al., 2009; Patzelt et al., 2011). As expected, the thiolated particles penetrated least well into the follicles, again attributed to their binding to keratin of the hair. PEGylation of these particles enhanced their deposition into the follicles compared to the parent thiolated materials with the PEG 750 nanoparticles penetrating to a mean maximal depth of $450 \mu \mathrm{m}$, again in the infundibulum above the sebaceous gland. However, the particles decorated with larger molecular weight PEG penetrated deeper, to $1400 \mu \mathrm{m}$ and close to the bulge region of the hair follicle (Vogt et al., 2007).

Others have similarly demonstrated the value of PEGylation for enhancing drug delivery. A silica core/PEG shell nanocomposite promoted uptake into MCF-7 breast cancer cells (Slowing et al., 2007). Liposomes loaded with calcipotriol were coated with variable concentrations of PEG to enhance their stability and increased penetration into the skin in comparison to non-PEGylated counterparts. Whilst PEG increased hydration of the stratum corneum by binding to water molecules resulting in 
enhanced permeability of the stratum corneum, variability in penetration of PEG coated calcipotriol liposomes was also attributed to the PEG conformation on the liposome surface (Knudsen et al., 2012). Deposition of gold nanorods decorated with PEG has also been studied in skin (Mahmoud et al., 2017) and were shown to penetrate into the follicular compartments but to a lesser extent than hydrophobic gold nanorods. However, after 24 hours, accumulation of PEGylated gold nanorods in deeper skin layers was significantly higher than for other gold nanorod systems with the authors suggesting that trans-follicular diffusion was facilitated by the polymers hydrophilic properties.

Our results illustrate that deposition into hair follicles is not solely by passive diffusion. Both the free dye and the PEGylated particles are hydrophilic and so diffusion theory would predict that the small molecular weight free drug would diffuse considerably faster than the nanoparticles. Penetration of the particles into the hair follicles is stimulated by movement of the hair, achieved by massaging the tissue in this study. The geared pump effect from hair shaft movement, described earlier, can carry particles deep within the follicle and it is notable that our largest nanoparticles, with PEG 5000 (89 nm diameter), penetrated deeper than the smaller PEG 750 material (79 nm diameter); it was suggested that particles closest in size to the hair cuticles $\sim 500 \mathrm{~nm}$ ) would be driven deepest into the follicles (Lademann et al., 2009; Patzelt et al., 2011). Further, upwards flow of sebum secreted from sebaceous gland can act as both a physical and chemical barrier for nanoparticle diffusion (Knorr et al., 2009; Lademann et al., 2009). Though this study used excised skin, it is likely that residual sebum within the follicle provided an additional hydrophobic barrier to penetration of the dye and particles.

The significantly different penetration depths of the two PEGylated particles are unlikely to result solely from the differences in their sizes. We have previously studied the diffusion of these nanoparticles in aqueous polymer solutions and water (Mun et al., 2014a). As expected, factors affecting diffusion of the nanoparticles were their size, the medium viscosity and the presence of interactions between the nanoparticles and the medium components. PEGylating nanoparticles enhanced their diffusivity in pure water, despite the size increase caused by functionalization, and this was attributed to the lubricating effect of PEG shell. PEGylation also reduced nanoparticle mucoadhesion to various tissues including the eye, the urinary bladder and nasal mucosa, again due a reduction in the exposed thiol groups on the particle surface which reduced binding, as seen here (Irmukhametova et al., 2011; Mun et al., 2014b; Ways et al., 2018). With reduced binding capacity, these particles also penetrated deeper in tissue. It was reported that PEG $<2000 \mathrm{Da}$ forms short chains with loss of flexibility compared to when molecular weight is > $2000 \mathrm{Da}$ (Owens and Peppas, 2006). In our studies, PEG forms a soft shell on the surface of the nanoparticles which reduces the number of thiol groups exposed to bind with keratin and so facilitates particle penetration into the hair follicles. Moreover, deeper penetration into the follicles of the PEGylated 5000 Da nanoparticles compared to 750 Da PEG particles is due to the greater flexibility and length of PEG chains which can further enclose and shield the thiol groups of the silica nanoparticles. 
This study has demonstrated that nanoparticles can be designed to penetrate to defined depths within hair follicles. Thiolated nanoparticles bind to keratin and are localized on the skin surface and near to the skin surface in the hair follicle. When decorated with polyethylene glycol, approximately half the thiol groups are removed from the particle surface and so binding to keratin is greatly reduced. Nanoparticles functionalized with higher molecular weight PEG (5000 Da) penetrated deeper into the follicles, to near the bulge region, than those with lower molecular weight PEG (750 Da) on their surface. The longer PAG chains are more flexible than their shorter counterparts and are better able to shield the remaining thiol groups on the particle surface and so allows deeper penetration by reducing particle-tissue binding. By manipulating the length of the PEG chain, it is feasible to targeted drug delivery to regions of the hair follicle.

\section{Acknowledgements:}

We are thankful to Ministry of Higher Education, Research and Innovation, Sultanate of Oman, for a PhD scholarship for Jamila Al Mahrooqi. We acknowledge the assistance of staff at Chemical Analysis Facility (CAF) and SBS Research Microscopy Facilities, University of Reading in fluorescence spectroscopy and fluorescence microscopy. We are grateful to Dr. Mark Henstock, The Pirbright Institute for providing pig skin used in this research.

\section{References:}

Al Mahrooqi, J.H., Mun, E.A., Williams, A.C., Khutoryanskiy, V. V., 2018. Controlling the size of thiolated organosilica nanoparticles. Langmuir. 34, 8347-8354. https://doi.org/10.1021/acs.langmuir.8b01556

Barry, B.W., Edwards, H.G.M., Williams, A.C., 1992. Fourier transform Raman and infrared vibrational study of human skin: Assignment of spectral bands. J. Raman Spectrosc. 23, 641-645. https://doi.org/10.1002/jrs.1250231113

Bragulla, H.H., Homberger, D.G., 2009. Structure and functions of keratin proteins in simple, stratified, keratinized and cornified epithelia. Journal of Anatomy. 214, 516-559. https://doi.org/10.1111/j.1469-7580.2009.01066.x

Brown, M.B., Williams, A.C., 2019. The Art and Science of Dermal Formulation Development. CRC Press, Florida. ISBN: 9780429059872. https://doi.org/10.1201/9780429059872

Connor, M.J., Wheeler, L.A., 1987. Depletion of cutaneous glutathione by ultraviolet radiation. Photochem. Photobiol. 46, 239-245. https://doi.org/10.1111/j.17511097.1987.tb04762.x

Cruz, C.F., Martins, M., Egipto, J., Osório, H., Ribeiro, A., Cavaco-Paulo, A., 2017. Changing the shape of hair with keratin peptides. RSC Adv. 7, 51581-51592. https://doi.org/10.1039/c7ra10461h

Cui, Y., Dong, H., Cai, X., Wang, D., Li, Y., 2012. Mesoporous silica nanoparticles capped with disulfide-linked PEG gatekeepers for glutathione-mediated controlled release. ACS Appl. Mater. Interfaces. 4, 3177-3183. 
https://doi.org/10.1021/am3005225

Doura, T., Nishio, T., Tamanoi, F., Nakamura, M., 2019. Relationship between the glutathione-responsive degradability of thiol-organosilica nanoparticles and the chemical structures. J. Mater. Res. 34, 1266-1278.

https://doi.org/10.1557/jmr.2018.501

Glasgow, B.J., 2016. Fluorescence lifetime imaging microscopy reveals quenching of fluorescein within corneal epithelium. Exp. Eye Res. 147, 12-19. https://doi.org/10.1016/j.exer.2016.04.008

Gniadecka, M., Nielsen, O.F., Christensen, D.H., Wulf, H.C., 1998. Structure of water, proteins, and lipids in intact human skin, hair, and nail. J. Invest. Dermatol. 110, 393-398. https://doi.org/10.1046/j.1523-1747.1998.00146.x

Hayashi, K., Maruhashi, T., Nakamura, M., Sakamoto, W., Yogo, T., 2016. One-pot synthesis of dual stimulus-responsive degradable hollow hybrid nanoparticles for image-guided trimodal therapy. Adv. Funct. Mater. 26: 8613-8622. https://doi.org/10.1002/adfm.201603394

Herkenne, C., Alberti, I., Naik, A., Kalia, Y.N., Mathy, F.X., Préat, V., Guy, R.H., 2008. In vivo methods for the assessment of topical drug bioavailability. Pharm. Res. 25, 87-103. https://doi.org/10.1007/s11095-007-9429-7

Higo, N., Naik, A., Bommannan, D.B., Potts, R.O., Guy, R.H., 1993. Validation of reflectance infrared spectroscopy as a quantitative method to measure percutaneous absorption in vivo. Pharm. Res. 10, 1500-1506. https://doi.org/10.1023/A:1018987612155

Irmukhametova, G.S., Mun, G.A., Khutoryanskiy, V. V, 2011. Thiolated mucoadhesive and PEGylated nonmucoadhesive organosilica nanoparticles from 3-mercaptopropyltrimethoxysilane. Langmuir. 27, 9551-9556. https://doi.org/10.1021/la201385h

Jain, B., Singh, B., Katare, O.P., Vyas, S.P., 2010. Development and characterization of minoxidil-loaded liposomal system for delivery to pilosebaceous units. J. Liposome Res. 20, 105-114. https://doi.org/ 10.1080/08982100903161449.

Jensen, L.B., Petersson, K., Nielsen, H.M., 2011. In vitro penetration properties of solid lipid nanoparticles in intact and barrier-impaired skin. Eur. J. Pharm. Biopharm. 79, 68-75. https://doi.org/10.1016/j.ejpb.2011.05.012

Klang, V., Schwarz, J.C., Hartl, A., Valenta, C., 2011. Facilitating in vitro tape stripping: Application of infrared densitometry for quantification of porcine stratum corneum proteins. Skin Pharmacol. Physiol. 24, 256-268. https://doi.org/10.1159/000326072

Knorr, F., Lademann, J., Patzelt, A., Sterry, W., Blume-Peytavi, U., Vogt, A., 2009. Follicular transport route - Research progress and future perspectives. Eur. J. Pharm. Biopharm. 71, 173-180. https://doi.org/10.1016/j.ejpb.2008.11.001

Knudsen, N.Ø., Rønholt, S., Salte, R.D., Jorgensen, L., Thormann, T., Basse, L.H., Hansen, J., Frokjaer, S., Foged, C., 2012. Calcipotriol delivery into the skin with PEGylated liposomes. Eur. J. Pharm. Biopharm. 81, 532-539. https://doi.org/10.1016/j.ejpb.2012.04.005

Kraeling, M.E.K., Topping, V.D., Keltner, Z.M., Belgrave, K.R., Bailey, K.D., Gao, X., Yourick, J.J., 2018. In vitro percutaneous penetration of silver nanoparticles in pig and human skin. Regul. Toxicol. Pharmacol. 95, 314-322. 
https://doi.org/10.1016/j.yrtph.2018.04.006

Küchler, S., Abdel-Mottaleb, M., Lamprecht, A., Radowski, M.R., Haag, R., SchäferKorting, M., 2009. Influence of nanocarrier type and size on skin delivery of hydrophilic agents. Int. J. Pharm. 377, 169-172.

https://doi.org/10.1016/j.ijpharm.2009.04.046

Kumar, P., Singh, S., Handa, V., Kathuria, H., 2018. Oleic acid nanovesicles of minoxidil for enhanced follicular delivery. Medicines. 5, 103-118. https://doi.org/10.3390/medicines5030103

Labouta, H.I., El-Khordagui, L.K., Kraus, T., Schneider, M., 2011a. Mechanism and determinants of nanoparticle penetration through human skin. Nanoscale. 3, 4989-4999. https://doi.org/10.1039/c1nr11109d

Labouta, H.I., Kraus, T., El-Khordagui, L.K., Schneider, M., 2011b. Combined multiphoton imaging-pixel analysis for semiquantitation of skin penetration of gold nanoparticles. Int. J. Pharm. 413, 279-282. https://doi.org/10.1016/j.ijpharm.2011.03.067

Labouta, H.I., Schneider, M., 2013. Interaction of inorganic nanoparticles with the skin barrier: Current status and critical review. Nanomedicine Nanotechnology, Biol. Med. 9, 39-54. https://doi.org/10.1016/j.nano.2012.04.004

Lademann, J., Patzelt, A., Richter, H., Antoniou, C., Sterry, W., Knorr, F., 2009. Determination of the cuticula thickness of human and porcine hairs and their potential influence on the penetration of nanoparticles into the hair follicles. J. Biomed. Opt. 14, 021014. https://doi.org/10.1117/1.3078813

Lademann, J., Richter, H., Schanzer, S., Knorr, F., Meinke, M., Sterry, W., Patzelt, A., 2011. Penetration and storage of particles in human skin: Perspectives and safety aspects. Eur. J. Pharm. Biopharm. 77, 465-468. https://doi.org/10.1016/j.ejpb.2010.10.015

Laffleur, F., Bernkop-Schnürch, A., 2018. Evaluation of dermal adhesive formulations for topical application. Eur. J. Pharm. Biopharm. 124, 89-94.

https://doi.org/10.1016/j.ejpb.2017.12.010

Larese, F.F., D’Agostin, F., Crosera, M., Adami, G., Renzi, N., Bovenzi, M., Maina, G., 2009. Human skin penetration of silver nanoparticles through intact and damaged skin. Toxicology. 255, 33-37. https://doi.org/10.1016/j.tox.2008.09.025

Lau, W.M., Heard, C.M., White, A.W., 2013. Design, synthesis and in vitro degradation of a novel co-drug for the treatment of psoriasis. Pharmaceutics.

5, 232-245. https://doi.org/10.3390/pharmaceutics5020232

Lenn, J.D., Neil, J., Donahue, C., Demock, K., Tibbetts, C.V., Cote-Sierra, J., Smith, S.H., Rubenstein, D., Therrien, J.P., Pendergrast, P.S., Killough, J., Brown, M.B., Williams, A.C., 2018. RNA Aptamer Delivery through Intact Human Skin. J. Invest. Dermatol. 138, 282-290. https://doi.org/10.1016/j.jid.2017.07.851

Mahmoud, N.N., Alkilany, A.M., Dietrich, D., Karst, U., Al-Bakri, A.G., Khalil, E.A., 2017. Preferential accumulation of gold nanorods into human skin hair follicles: Effect of nanoparticle surface chemistry. J. Colloid Interface Sci. 503, 95-102. https://doi.org/10.1016/j.jcis.2017.05.011

Mathes, C., Melero, A., Conrad, P., Vogt, T., Rigo, L., Selzer, D., Prado, W.A., De Rossi, C., Garrigues, T.M., Hansen, S., Guterres, S.S., Pohlmann, A.R., Beck, R.C.R., Lehr, C.M., Schaefer, U.F., 2016. Nanocarriers for optimizing the 
balance between interfollicular permeation and follicular uptake of topically applied clobetasol to minimize adverse effects. J. Control. Release. 223, 207214. https://doi.org/10.1016/j.jconrel.2015.12.010

Mun, E. A., Hannell, C., Rogers, S.E., Hole, P., Williams, A.C., Khutoryanskiy, V. V., 2014a. On the role of specific interactions in the diffusion of nanoparticles in aqueous polymer solutions. Langmuir. 30, 308-317. https://doi.org/10.1021/la4029035

Mun, E A., Morrison, P.W.J., Williams, A.C., Khutoryanskiy, V. V., 2014b. On the barrier properties of the cornea: A microscopy study of the penetration of fluorescently labeled nanoparticles, polymers, and sodium fluorescein. Mol. Pharm. 11, 3556-3564. https://doi.org/10.1021/mp500332m

Mun, E.A., Williams, A.C., Khutoryanskiy, V. V, 2016. Adhesion of thiolated silica nanoparticles to urinary bladder mucosa: Effects of PEGylation, thiol content and particle size. Int. J. Pharm. 512, 32-38.

https://doi.org/10.1016/j.ijpharm.2016.08.026

Nagelreiter, C., Mahrhauser, D., Wiatschka, K., Skipiol, S., Valenta, C., 2015. Importance of a suitable working protocol for tape stripping experiments on porcine ear skin: Influence of lipophilic formulations and strip adhesion impairment. Int. J. Pharm. 491, 162-169. https://doi.org/10.1016/j.ijpharm.2015.06.031

Otberg, N., Richter, H., Schaefer, H., Blume-Peytavi, U., Sterry, W., Lademann, J., 2004. Variations of hair follicle size and distribution in different body sites. J. Invest. Dermatol. 122, 14-19. https://doi.org/10.1046/j.0022202X.2003.22110.x

Owens, D.E., Peppas, N.A., 2006. Opsonization, biodistribution, and pharmacokinetics of polymeric nanoparticles. Int. J. Pharm. 307, 93-102. https://doi.org/10.1016/j.ijpharm.2005.10.010

Padois, K., Cantiéni, C., Bertholle, V., Bardel, C., Pirot, F. Falson, F. 2011. Solid lipid nanoparticles suspension versus commercial solutions for dermal delivery of minoxidil. Int. J. Pharm., 416, 300-304. https://doi.org/10.1016/j.ijpharm.2011.06.014

Partenhauser, A., Zupančič, O., Rohrer, J., Bonengel, S., Bernkop-Schnürch, A., 2016. Thiolated silicone oils as adhesive skin protectants for improved barrier function. Int. J. Cosmet. Sci. 38, 257-265. https://doi.org/10.1111/ics.12284

Patzelt, A., Richter, H., Knorr, F., Schäfer, U., Lehr, C.M., Dähne, L., Sterry, W., Lademann, J., 2011. Selective follicular targeting by modification of the particle sizes. J. Control. Release. 150, 45-48. https://doi.org/10.1016/j.jconrel.2010.11.015

Pensado, A., Chiu, W.S., Cordery, S.F., Rantou, E., Bunge, A.L., Delgado-Charro, M.B., Guy, R.H., 2019. Stratum corneum sampling to assess bioequivalence between topical acyclovir products. Pharm. Res. 36, 180-196. https://doi.org/10.1007/s11095-019-2707-3

Porfiryeva, N.N., Moustafine, R.I., Khutoryanskiy, V. V., 2020. PEGylated systems in pharmaceutics. Polym. Sci. Ser. C. 62, 62-74. https://doi.org/10.1134/S181123822001004X

Prasetyanto, E.A., Bertucci, A., Septiadi, D., Corradini, R., Castro-Hartmann, P., De Cola, L., 2016. Breakable hybrid organosilica nanocapsules for protein delivery. Angew. Chemie Int. Ed. 55, 3323-3327. 
https://doi.org/10.1002/anie.201508288

Raber, A.S., Mittal, A., Schäfer, J., Bakowsky, U., Reichrath, J., Vogt, T., Schaefer, U.F., Hansen, S., Lehr, C.M., 2014. Quantification of nanoparticle uptake into hair follicles in pig ear and human forearm. J. Control. Release. 179, 25-32. https://doi.org/10.1016/j.jconrel.2014.01.018

Rangsimawong, W., Opanasopit, P., Rojanarata, T., Duangjit, S., Ngawhirunpat, T., 2016. Skin transport of hydrophilic compound-loaded PEGylated lipid nanocarriers: Comparative study of liposomes, niosomes, and solid lipid nanoparticles. Biol. Pharm. Bull. 39, 1254-1262.

https://doi.org/10.1248/bpb.b15-00981

Roque, L.V., Dias, I.S., Cruz, N., Rebelo, A., Roberto, A., Rijo, P., Reis, C.P., Design of finasteride-loaded nanoparticles for potential treatment of alopecia. Skin Pharmacol. Physiol., 30, 197-204. https://doi.org/10.1159/000475473

Russell, L.M., Guy, R.H., 2012. Novel imaging method to quantify stratum corneum in dermatopharmacokinetic studies: Proof-of-concept with acyclovir formulations. Pharm. Res. 29, 3362-3372. https://doi.org/10.1007/s11095012-0831-4

Salim, S., Kamalasanan, K., 2020. Controlled drug delivery for alopecia: A review. J. Control. Release. 325, 84-99. https://doi.org/10.1016/j.jconrel.2020.06.019

Schiedel, C., Melero, A., Conrad, P., Vogt, T., Rigo, L., Selzer, D., Prado, W.A., De Rossi, C., Garrigues, T., Hansen, S., Guterres, S.S., Pohlmann, A., Beck, R., Lehr, C-M., Schaefer, U. 2015. Nanocarriers for optimizing the balance between interfollicular permeation and follicular uptake of topically applied clobetasol to minimize adverse effects. J. Control. Release. 223, 207-214. https://doi.org/10.1016/j.jconrel.2015.12.010.

Slowing, I.I., Trewyn, B.G., Giri, S., Lin, V.S.Y., 2007. Mesoporous silica nanoparticles for drug delivery and biosensing applications. Adv. Funct. Mater. 17, 1225-1236. https://doi.org/10.1002/adfm.200601191

Song, L., Hennink, E.J., Young, I.T., Tanke, H.J., 1995. Photobleaching kinetics of fluorescein in quantitative fluorescence microscopy. Biophys. J. 68, 25882600. https://doi.org/10.1016/S0006-3495(95)80442-X

Takeuchi, I., Hida, Y., Makino K., 2018. Minoxidil-encapsulated poly(L-lactide-co glycolide) nanoparticles with hair follicle delivery properties prepared using W/O/W solvent evaporation and sonication. Biomed. Mater. Eng. 29, 217228. https://doi.org/10.3233/BME-171724

Teichmann, A., Jacobi, U., Ossadnik, M., Richter, H., Koch, S., Sterry, W., Lademann, J., 2005. Differential stripping: Determination of the amount of topically applied substances penetrated into the hair follicles. J. Invest. Dermatol. 125, 264-269. https://doi.org/10.1111/j.0022-202X.2005.23779.x

Vogt, A., Combadiere, B., Hadam, S., Stieler, K.M., Lademann, J., Schaefer, H., Autran, B., Sterry, W., Blume-Peytavi, U., 2006. $40 \mathrm{~nm}$, but not 750 or 1,500 nm, nanoparticles enter epidermal CD1a+ cells after transcutaneous application on human skin. J. Invest. Dermatol. 126, 1316-1322. https://doi.org/10.1038/sj.jid.5700226

Vogt, A., Hadam, S., Heiderhoff, M., Audring, H., Lademann, J., Sterry, W., BlumePeytavi, U., 2007. Morphometry of human terminal and vellus hair follicles. Exp. Dermatol. 16, 946-950. https://doi.org/10.1111/j.1600-0625.2007.00602.x 
Vogt, A., Wischke, C., Neffe, A.T., Ma, N., Alexiev, U., Lendlein, A., 2016. Nanocarriers for drug delivery into and through the skin - Do existing technologies match clinical challenges? J. Control. Release. 242, 3-15. https://doi.org/10.1016/j.jconrel.2016.07.027

Wang, Y.Y., Lai, S.K., Suk, J.S., Pace, A., Cone, R., Hanes, J., 2008. Addressing the PEG mucoadhesivity paradox to engineer nanoparticles that "slip" through the human mucus barrier. Angew. Chemie Int. Ed. 47, 9726-9729. https://doi.org/10.1002/anie.200803526

Ways, T.M.M., Lau, W.M., Ng, K.W., Khutoryanskiy, V. V., 2018. Synthesis of thiolated, PEGylated and POZylated silica nanoparticles and evaluation of their retention on rat intestinal mucosa in vitro. Eur. J. Pharm. Sci. 122, 230-238. https://doi.org/10.1016/J.EJPS.2018.06.032

Williams, A.C., Edwards, H.G.M., Barry, B.W., 1994. Raman spectra of human keratotic biopolymers: Skin, callus, hair and nail. J. Raman Spectrosc. 25, 95-98. https://doi.org/10.1002/jrs.1250250113

Wu, X., Biatry, B., Cazeneuve, C., Guy, R.H., 2009. Drug delivery to the skin from sub-micron polymeric particle formulations: Influence of particle size and polymer hydrophobicity. Pharm. Res. 26, 1995-2001. 10.1007/s11095-0099915-1

Yang, Y., Wan, J., Niu, Y., Gu, Z., Zhang, J., Yu, M., Yu, C., 2016. StructureDependent and glutathione-responsive biodegradable dendritic mesoporous organosilica nanoparticles for safe protein delivery. Chem. Mater. 28, 90089016. https://doi.org/10.1021/acs.chemmater.6b03896

Zhang, L.W., Yu, W.W., Colvin, V.L., Monteiro-Riviere, N.A., 2008. Biological interactions of quantum dot nanoparticles in skin and in human epidermal keratinocytes. Toxicol. Appl. Pharmacol. 228, 200-211.

https://doi.org/10.1016/j.taap.2007.12.022

Zhang, Q., Liu, F., Nguyen, K.T., Ma, X., Wang, X., Xing, B., Zhao, Y., 2012. Multifunctional mesoporous silica nanoparticles for cancer-targeted and controlled drug delivery. Adv. Funct. Mater. 22, 5144-5156. https://doi.org/10.1002/adfm.201201316

Zhou, M., Du, X., Li, W., Li, X., Huang, H., Liao, Q., Shi, B., Zhang, X., Zhang, M., 2017. One-pot synthesis of redox-triggered biodegradable hybrid nanocapsules with a disulfide-bridged silsesquioxane framework for promising drug delivery. J. Mater. Chem. B. 5, 4455-4469. https://doi.org/10.1039/c6tb03368g 
